\title{
Wireless calibration for Industry 4.0
}

\author{
Roberto Benitez Mr., ${ }^{1, *}$, and Roberto Benitez $\mathrm{Jr}^{2}$ \\ ${ }^{1}$ ETALONS, General Director, S. del Fraile 117, A. Seco, Monterrey, N.L. México \\ ${ }^{2}$ ETALONS, Commercial Manager, S. del Fraile 117, A. Seco, Monterrey, N.L. México
}

\begin{abstract}
During the 1980 's, before the industrial globalization took place, some recommended practices or standards were used to provide the calibration services for inspection, measurement and testing equipment (IM\&TE) in America. Before the ISO Standards were used to provide quality services or to be ISO accredited, the calibration laboratories were using the MIL-STD-45662A. While the ISO standards became the most applicable references for quality systems certification and for the accreditation of the calibration laboratories the ISO/IEC-17025 became the standard that should be met for the accreditation. Since the beginning of the calibration services, the calibration certificates contained information about the status of the instrument, depending of its type and the method used to calibrate the instrument, also some other requirements were included in the certificates, like the uncertainty and the evidence of the proficiency tests that the laboratory had to provide. With the technological advancement in electronics, the instruments became more sophisticated and accurate. The use of computers and dedicated software for calibration developed new calibration methods. Industry 4.0 includes a wide variety of sensors to monitor different parameters along the process or to measure quality specifications, most of those sensors are electronically integrated to the process control. Metrology 4.0 is giving a new way of calibration services applying the AI and IoT.
\end{abstract}

\section{Introduction}

When industry globalization took place around the 1980's, the manufacturing process began to be certified by quality standards. The International Standardization Organization (ISO) issued the standards to be certified the quality systems for manufacturing process, services companies and calibration laboratories. One of the requirements for the process certification is the calibration of the inspection, measurement and testing equipment. Metrology, the measuring science, stablishes the regulation for the instruments calibration based in an International System of Units (SI). Since the beginning of the calibration services, the calibration certificates contain information about the status of the instrument, depending of its type and the method used to calibrate the instrument. With the technological advance, the instruments became more sophisticated and accurate. In the past, older measuring instruments were calibrated by analogic or mechanic reference standards. Industry 4.0 includes a wide variety of sensors used to measure quality specifications, most

\footnotetext{
* Corresponding author: roberto@etalons.com.mx
} 
of those sensors are electronically integrated to the process control or in some cases their indications are send by wireless signal. In order to calibrate the electronic or digital sensors, it is necessary to use digital or electronic reference standards. Nowadays, some instrument manufacturers are providing new reference standards to meet the requirements to calibrate the sensors for Industry 4.0. The recent redefinition of the SI, and the technological advance, permits to develop more accurate reference standards to calibrate the instruments in the manufacturing process. The use of the Internet of Things (IoT) in the calibration process is more frequently applied. The International Metrology Vocabulary (VIM) defines calibration as: "operation that under specified conditions, in a first step, stablishes a relation between the quantity values with measurement uncertainties provided by measurement standards and corresponding indications with associated measurement uncertainties and, in a second step, uses this information to stablish a relation for obtaining a measurement result from an indication", this operation should be made according to a specific method or a calibration procedure, most of the times as an international recommendation. One of the ISO quality standards requirements is that "where necessary to ensure valid results, measuring equipment shall be calibrated, at specified interval, against measurement standards". The calibration result should be documented in a Calibration Certificate. During the 1980 's, the calibration laboratories provided calibration certificates focused on the traceability of the measurements by making references through primary laboratory report number. At that time, most of the instruments were sent to the calibration laboratory that in some cases was far away of the plant or in another department of the factory. With the development of ISO standards, the calibration requirements were more complete, and more calibration data was included in the calibration certificates.

\section{Calibration requirements}

The measurement uncertainty estimation and the technical competence of the calibration technician are some of the ISO requirements for an instrument calibration. Also, the ISO standards required that the calibration laboratory should be ISO accredited. Another requirement of the ISO quality standards was the accreditation of the calibration service providers [2]. When the industrial globalization took place and the computers were introduced for administration activities and automation of the production process, the quality systems required to maintain the "records of the calibration activity for all gauges, measuring and test equipment, needed to provide evidence of conformity of product to determined requirements".

\subsection{Former type instruments}

Before the electronic era, most of the measuring instruments were based in complicated mechanisms or passive elements, as an example, we can mention the gas operated thermometers. This type of instruments was complicated to remove in order to send them for calibration, making to expensive the calibration service and this process takes to much time to be performed. The calibration service provider for mechanical and analogic measuring instruments built big facilities to keep the reference standards that most of the times were large equipment, and those facilities were also far away from the process plant. Only few factories developed their own calibration laboratories having a limited scope that did not cover all their measuring instruments. Even more, the laboratories of the calibration service providers should be ISO accredited. 


\subsection{Electronic or digital measuring instruments}

Nowadays, the smart factory uses electronic or digital sensors (Fig. 1) to measure the different parameters in their manufacturing process.

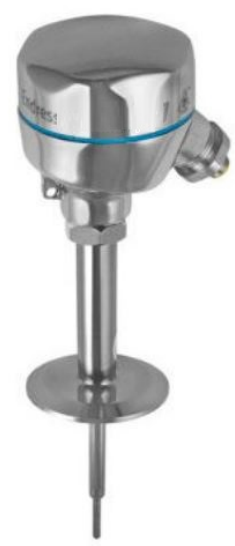

Figure 1. Smart Temperature sensor

Everyday new smart sensors are developed, and mobile applications (App's) are used to control or to monitor the manufacturing process. One of the current applications of the Metrology 4.0 is in the brewery industry (Fig. 6). In order to monitor and control the process and the conditions of the product, since the incoming material up to the final product, it is necessary to have different types of instruments; weighing devices, thermometers, pressure gauges, flow meters, level meters, and some many others. Most of the time, these sensors send the information to the main panel control via wire or wireless. The information that comes from the smart sensors is presented in the control panel, and it could be send to a corporate computer or to a mobile device, by this way, the information shown in the control panel, could be sed to any place in the same plant or to some other facilities far away, even facilities that are located around the world. Even though the smart sensors are accurate measurement instruments, those sensors should be calibrated in order to ensure valid results, at specific intervals, and against measurement standards traceable to the SI.

\section{Calibration methods}

\subsection{Traditional calibration method}

The traditional method to calibrate temperature sensors consists in a Dry Well calibrator with an integrated temperature indicator, the control panel of the process (system to be calibrated), radiofrequency mobile devices or cellular phone, two calibration technicians and the use of a text processor. The Dry Well calibrator should be connected to the power outlet, most of the times by a power cord extension. The control panel is the screen where the operator monitors the process temperatures. 


\section{Procedure}

1.- Two calibration technicians are set in place, technician A next to the sensor under calibration, technician B in the control panel.

2.- Technician A looks for the nearest power outlet to connect the calibrator and set the first temperature value of calibration.

3.- Technician A introduces the temperature sensor in the Dry Well calibrator.

4.- Technician B receives via radiofrequency or mobile phone call the temperature value of the Dry Well and takes the temperature value of the sensor shown in the control panel.

5.- Technician $B$ writes down both values in a text processor.

6.- Technician A sets the second temperature value of calibration and gives the value shown in the calibrator to Technician B.

7.- Technician $B$ writes down both values and continue in the same way for all other values of calibration.

8.- At the end of the process the Calibration Certificate is issued and sent to the customer as an electronic document.

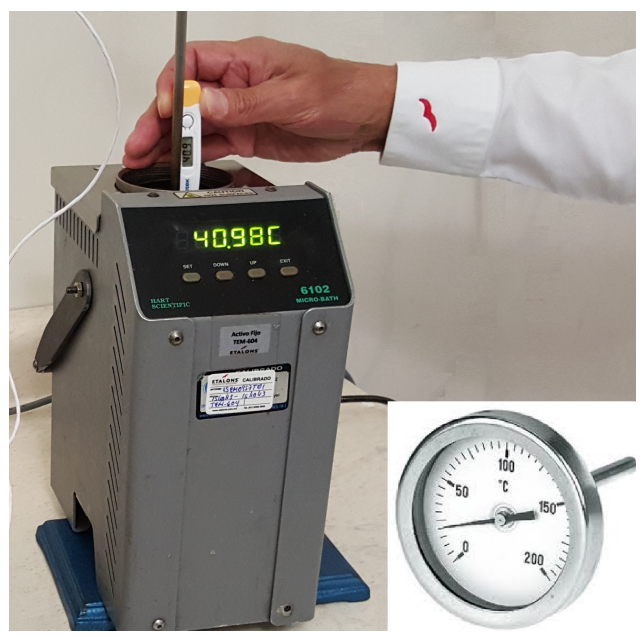

Figure 2. Former Temperature Calibration Standard

Some disadvantages of this method are the necessity to have two calibration technicians to calibrate each temperature sensor, that means that this procedure is expensive and time consuming, also it is quite difficult to have an electrical cord connected to the power outlet that can cause an accident or electrical shock. Another disadvantage is the possibility to make errors during the data transcription or during the verbal communication through radios or mobile call.

\subsection{Wireless Calibration System}

Being in industrial calibration services for more than 39 years, three years ago we found the necessity to calibrate temperature measuring sensors in a more efficient way. With the use of traditional standards to calibrate temperature sensors, we needed to move the Dry Well Standard that had to be connected to the power outlet (Fig. 2). According to the calibration procedure for temperature sensors, it is necessary to place the sensor inside of the Dry Well and then compare the indicated temperature of both instruments. Most of the times, the control panel where process sensor temperature is displayed is located at a great distance 
from the Dry Well calibrator, reason why the two-way communication via radio or mobile phone is necessary to read and to register the indicated values. This process requires two calibration technicians to be performed making quite expensive the calibration service. Analyzing the issues this could cost, we designed an equipment that could improve the results and provide a more efficient calibration method. The first step we did, was the integration of an autonomous power unit, formed by one dc battery, one dc-ac converter, one energy charger, and the Dry Well Calibrator (Fig. 4). After the power unit was assembled, a wireless Resistance Temperature Device (RTD) sensor was attached to the power unit. The RTD sensor has the capability to connect to the network and to have control of the temperature standard and the transmission of the data to the user or the calibration technician, a software application was developed for this purpose [5].

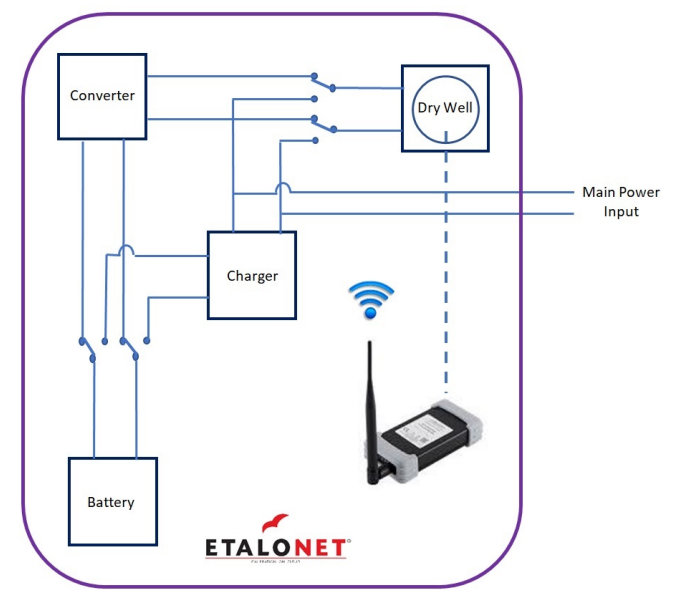

Figure 3. Smart Temperature Calibration system

With this system we calibrate the temperature sensors that are installed in some brewery plants in Mexico and we started using it to calibrate smart thermometers located at pharma warehouses.

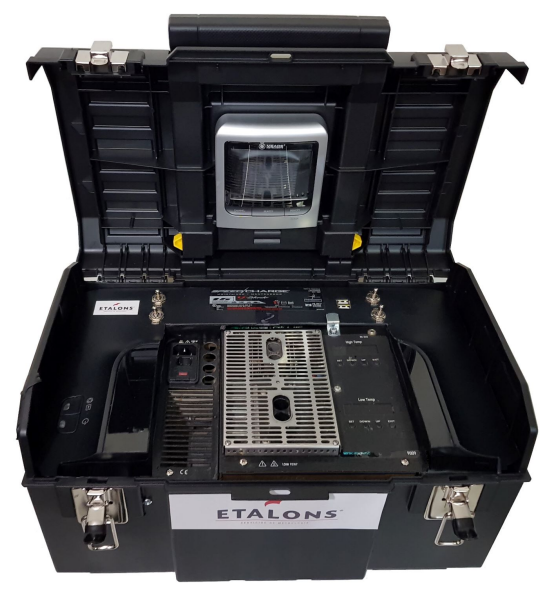

Figure 4. Smart Temperature Calibrator 
Smart factories are growing in different areas as automotive, healthcare, food and beverages, aeronautics and some others. Smart cities also will need to measure water supply, energy, gas, transportation, etc. All these measurements will need to be ensure for legal trade.

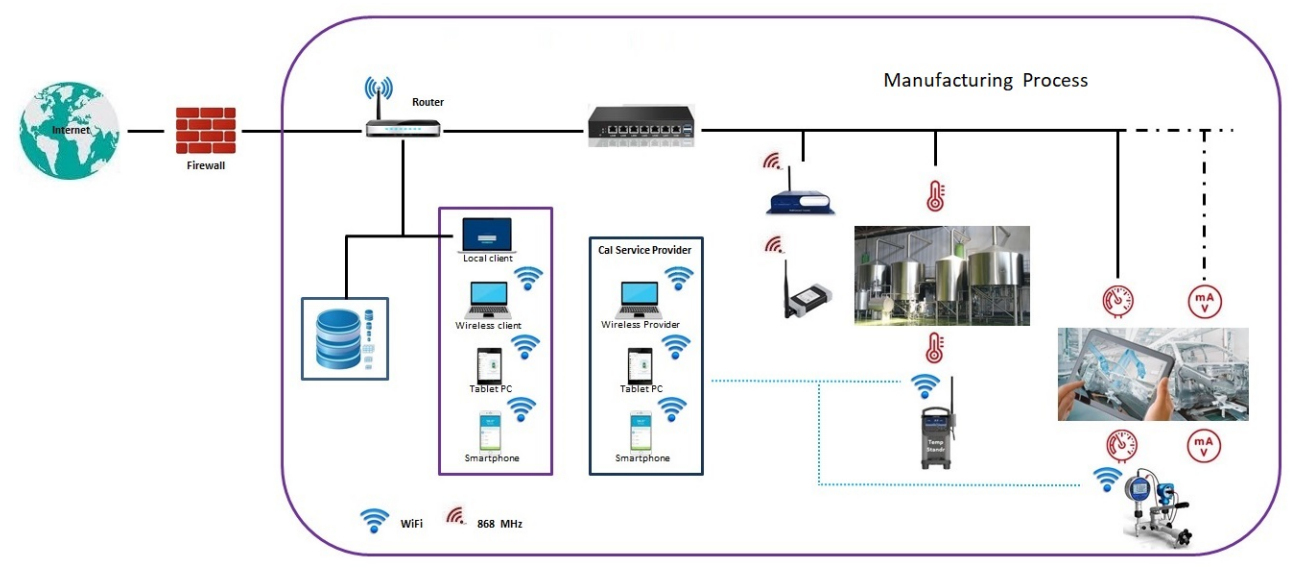

Figure 5. Smart factory concept

\section{Proposed calibration method}

The proposed calibration method to calibrate temperature sensors consists in a totally wireless Dry Well calibrator with an external Temperature Standard, a software application to transmit via wifi the temperature value to a mobile device. This system is operated by one calibration technician. The software application permits to send the calibration data automatically to the customer data base.

Procedure

1.- The calibration technician sets the sensor to be calibrated inside of the wireless Temperature Calibrator.

2.- The calibration technician sets the programed steps in the Dry Well for the values to be calibrated.

3.- The calibration technician moves to the process control panel and by using the software application starts to collect the values indicated in the control panel and the correspondent values shown in the mobile device.

4.- At the end of the process the calibration technician can send all the data directly to the customer data base.

The main advantages of this system are: to have a complete wireless temperature calibrator supported by rechargeable battery pack that keeps in operation for at least 12 hours before the next recharge is needed. This system is operated by only one calibration technician saving time and costs. No errors are introduced because the data is captured automatically by the software application program. Currently we have been using this system to provide calibration services to a brewery plant, and we were using the system for most than one year without any failure. 


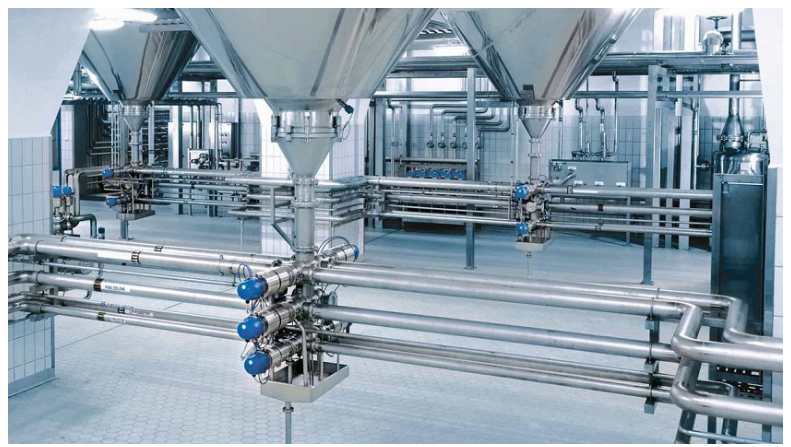

Figure 6. Smart industry

With these systems, it is possible to monitor every part of the manufacturing process from any place inside or outside of the process plant, even in some other part around the world. The smart sensors that the Industry 4.0 is using to monitor the process measurements, require new accurate and smart calibration standards. The temperature standards that we were using in the past, made the calibration of those sensors complicated, that was the reason why we developed a wireless on site temperature calibration system.

\section{Conclusion}

With the development of this wireless temperature calibrator (Fig. 4) we have the advantage that it is not necessary to be connected to any power outlet, not having losing cables on the floor, that means that the calibrator is perfect to verify sensors in difficult to reach places. With the two ways wifi communication between the Calibrator and the mobile smart phone (Fig. 5), the calibration technician can upload immediately the calibration results to the customer data system instead of the former procedure that has been taken more than two day to delivery to the customer. With this wireless on site calibration system, we demonstrate that the requirement of the measurement assurance program of the smart factories could be met with the application of the Metrology 4.0. In the future, with the use of quantum measuring units and the practical results of projects like "The NIST on a Chip", we will have reference standards integrated in small units. In ETALONS we are currently developing a wireless on site calibration standards for pressure and mass.

\section{References}

[1] BIPM JCGM 200:2012 International vocabulary of metrology - Basic and general concepts and associated terms (VIM) $3^{\text {rd }}$ edition.

[2] ISO/IEC 17025:2017 General requirements for the competence of testing and calibration laboratories Third edition 2017-11

[3] AUTOMOTIVE QUALITY MANAGEMENT SYSTEM STANDARD IATF 16949:2016 - Quality management system requirements for automotive production and relevant service parts organizations. Ist Edition, 1 October 2016.

[4] IEC 60050-300:2001, International Electrotechnical Vocabulary - Electrical and electronic measurements and measuring instruments - Part 311: General terms relating to measurements - Part 312: General terms relating to electrical measurements - Part 313: Types of electrical measuring instruments - Part 314: Specific terms according to the type of instrument.

[5] R. Benitez Mr, R. Benitez Jr, C. Ramirez, J. Vazquez, "Sensors calibration for Metrology 4.0" 2019 IEEE International Workshop on Metrology for Industry 4.0 and IoT, Naples, Italy, June 4-6, 2019, pp. 279-282. 